\title{
Two Fermions in a Double Well: Exploring a Fundamental Building Block of the Hubbard Model
}

Simon Murmann, ${ }^{*}$ Andrea Bergschneider, Vincent M. Klinkhamer, Gerhard Zürn, Thomas Lompe, ${ }^{\dagger}$ and Selim Jochim Physikalisches Institut der Universität Heidelberg, Im Neuenheimer Feld 226, 69120 Heidelberg, Germany

(Received 4 November 2014; published 23 February 2015)

\begin{abstract}
We have prepared two ultracold fermionic atoms in an isolated double-well potential and obtained full control over the quantum state of this system. In particular, we can independently control the interaction strength between the particles, their tunneling rate between the wells and the tilt of the potential. By introducing repulsive (attractive) interparticle interactions we have realized the two-particle analog of a Mott-insulating (charge-density-wave) state. We have also spectroscopically observed how second-order tunneling affects the energy of the system. This work realizes the first step of a bottom-up approach to deterministically create a single-site addressable realization of a ground-state Fermi-Hubbard system.
\end{abstract}

DOI: 10.1103/PhysRevLett.114.080402

PACS numbers: 05.30.Fk, 67.85.Lm, 71.10.Fd

In the presence of strong correlations, the understanding of quantum many-body systems can be exceedingly difficult. One way to simplify the description of such systems is to use a discrete model where the motion of the particles is restricted to hopping between the sites of a lattice. The paradigmatic example for this approach is the Hubbard model, which reduces the physics of a quantum many-body system to tunneling of particles between adjacent sites and interactions between particles occupying the same site. While this model captures essential properties of electrons in a crystalline solid and provides a microscopic explanation for the existence of Mott-insulating and antiferromagnetic phases, many questions about this Hamiltonian—such as whether it can explain $d$-wave superfluidity-are still unanswered [1].

A promising approach to answer these questions is to use ultracold atoms trapped in periodic potentials as quantum simulators of the Hubbard model [2-8]. Such experiments have been performed both in large- and small-scale systems. Degenerate gases loaded into optical lattices have been used to observe the transition to the bosonic $[9,10]$ and fermionic Mott insulator [3,4]. The first observation of second-order tunneling was achieved in a small-scale system by studying the tunneling dynamics of bosonic atoms in an array of separated double wells $[11,12]$. In a recent experiment, these two regimes have been connected by splitting a fermionic Mott insulator into individual double wells. In this way, the strength of the antiferromagnetic correlations in the many-body system could be determined by measuring the fraction of double wells with two atoms in the spin-singlet configuration [13]. But, despite the observation of antiferromagnetic correlations $[13,14]$, current experiments using fermionic atoms have so far failed to reach temperatures below the critical temperature of spin ordering $[15,16]$.

Recently, new experimental techniques have been developed which allow for the deterministic preparation of few-particle systems in the ground state of a single potential well [17-19]. This makes it feasible to use ultracold atoms to study many-body physics in a bottom-up approach, i.e., to start from the fundamental building block of the system and watch how many-body effects emerge as one gradually increases the system's size [20]. Here we report on the realization of the fundamental building block of the FermiHubbard model at half filling, which consists of one $|\uparrow\rangle$ and one $|\downarrow\rangle$ particle in a spin-singlet configuration in a doublewell potential.

By starting from this two-site realization of the Hubbard model, we can test our building block in a regime where the model can still be easily solved. In the Hubbard regime, the spatial wave function $|\Psi\rangle$ of this two-particle system can be written in the basis $\{|L L\rangle,|L R\rangle,|R L\rangle,|R R\rangle\}$. These basis states are all possible combinations of the localized singleparticle states $|L\rangle$ and $|R\rangle$ of one particle in the ground state of either the left or the right well. In this basis, the spatial part of the Hamiltonian is

$$
H=\left(\begin{array}{cccc}
U+2 \Delta & -J & -J & 0 \\
-J & 0 & 0 & -J \\
-J & 0 & 0 & -J \\
0 & -J & -J & U-2 \Delta
\end{array}\right)
$$

with the tunneling matrix element $J$, the on-site interaction energy $U$ and the energy tilt $2 \Delta$ between the wells. Diagonalizing this Hamiltonian leads to three eigenstates $(|a\rangle,|b\rangle$, and $|c\rangle)$ which are symmetric and one eigenstate $(|d\rangle)$ which is antisymmetric with respect to particle exchange [Fig. 1(b)].

To prepare our double-well system, we start with two ${ }^{6} \mathrm{Li}$ atoms in different hyperfine states in the motional ground state of a single optical microtrap [17] (Sec. I of the Supplemental Material [21]). We then slowly ramp on a second potential well and thereby deform our single trap 
(a)

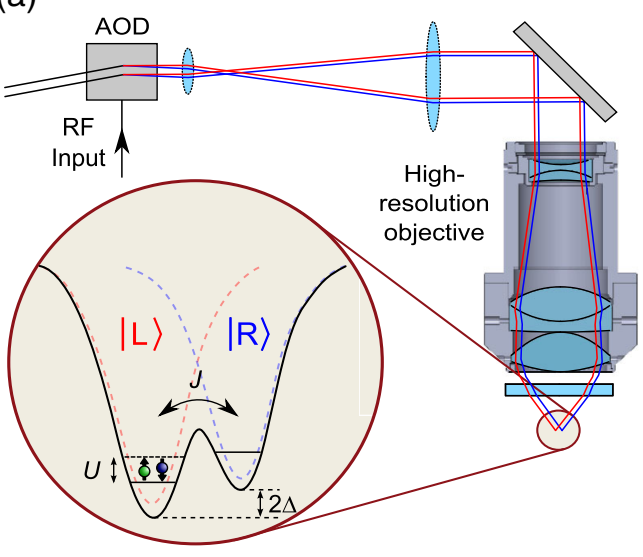

(c)

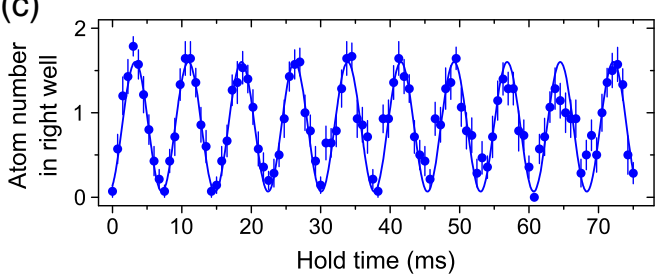

(b)

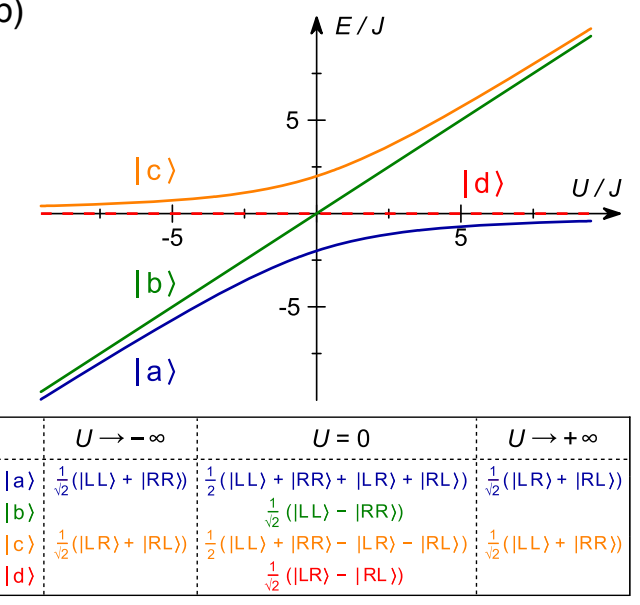

(d)

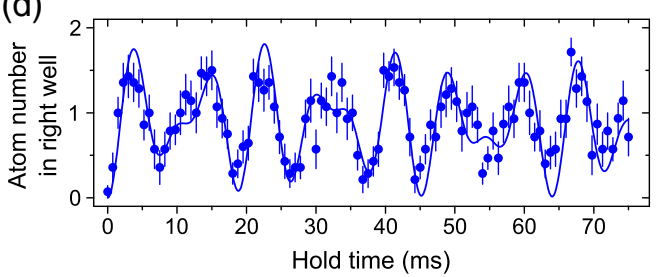

FIG. 1 (color online). Experimental realization and eigenenergies of the two-site Hubbard model. (a) Experimental setup: The doublewell potential is created by focusing two laser beams with a high-resolution objective. By independently controlling the intensity and position of the two laser beams with an acousto-optic deflector (AOD) we can tune the tunnel coupling $J$ and the tilt $\Delta$ between the two wells. (b) Energies of the four lowest two-particle eigenstates in a symmetric double-well potential as a function of the on-site interaction energy $U$. (c),(d) Tunneling of two particles in the double well. The data show the time evolution of the particle number in the right well after initializing the system with both particles in the left well and abruptly switching on the tunnel coupling $J$ between the two sites. For $U \approx 0$ and $\Delta \approx 0$ (c), we can extract the value of the tunnel coupling by fitting the data with a damped sine wave. For intermediate interaction strength $(U \approx J)(\mathrm{d})$, we observe correlated tunneling of the two particles, which shows good agreement with the prediction from the Hubbard model (solid line). The error bars denote the $1 \sigma$ statistical uncertainty.

into a double-well potential. During this process, we keep the coupling between the wells negligible and thus initialize the system in state $(|L L\rangle)$ where both atoms reside in the ground state of the left well. This state is the starting point for all our measurements. We can prepare it with a fidelity of more than $90 \%$. The predominant error is that there is only one atom in the trapping potential, while the probability to start with three atoms is $\lesssim 1 \%$.

Since the spatial wave function of the initial state is symmetric with respect to particle exchange, the spin wave function of the two fermions is in a singlet configuration. In the experiments presented here, we do not couple the position and the spin of the particles and therefore restrict the spatial wave function of the system to the symmetric eigenstates $|a\rangle,|b\rangle$, and $|c\rangle$.

In a first set of experiments, we study the tunneling dynamics of the two particles in our double-well potential to characterize the Hubbard parameters $J, U$, and $\Delta$ of our system. To do this, we initialize the system in state $|L L\rangle$ and abruptly reduce the height of the potential barrier, which allows the atoms to tunnel between the wells. To observe the resulting dynamics, we let the system evolve for different durations and then freeze the spatial distribution of the atoms by quickly increasing the barrier height. We then count the number of atoms in one of the wells by recapturing them into a magneto-optical trap and measuring their fluorescence [27] [17] (Sec. III of [21]).

For a noninteracting system and a small tilt of the double well $(|\Delta| \lesssim J)$, we observe long-lived tunneling oscillations whose frequencies we can set by tuning the barrier height (Sec. V.A, Fig. S1 and Fig. S2(a) of [21]). As an example, a typical oscillation for $\Delta \simeq 0$ with a frequency of $2 J / h \simeq$ $134 \mathrm{~Hz}$ is shown in Fig. 1(c). To calibrate $\Delta$, we measure the oscillation frequency for different tilts and find good agreement with the effective coupling strength $J_{\text {eff }}=\sqrt{J^{2}+\Delta^{2}}$ of a two-level system (Sec. V.B and Fig. S2(b) of [21]).

For an interacting system, the interaction energy creates an effective detuning for the tunneling of a single particle. In the limit of strong interactions and a symmetric double well $(U \gg J$ and $\Delta=0)$, the atoms can therefore only tunnel as pairs [28]. However, we can restore single-particle tunneling by setting a tilt that compensates for the interaction shift [11]. This allows us to calibrate the on-site interaction energy $U$ by measuring the strength of singleparticle tunneling as a function of the tilt $\Delta$ for different interaction strengths (Fig. 2). We find good agreement with 


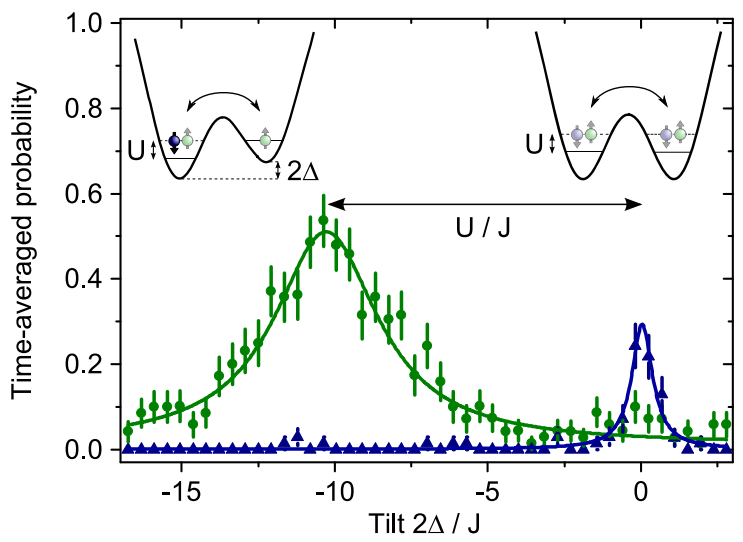

FIG. 2 (color online). Single-particle and pair tunneling as a function of the tilt $\Delta$ at an interaction energy of $U / J=$ $10.05 \pm 0.19$. The data points show the time-averaged probability of finding a single particle (green circles) or a pair of particles (blue triangles) in the right well after initializing the system with two atoms in the left well and switching on the tunneling. Pair tunneling is resonant in a symmetric double well, while conditional single-particle tunneling occurs for a tilt of $-2 \Delta=U$. The error bars denote the $1 \sigma$ statistical uncertainty.

a calculation of the interaction energy of two particles in the single well [24] (Sec. V.C and Fig. S3 of [21]).

Using the parameters determined from these measurements, the two-site Hubbard Hamiltonian [Eq. (1)] fully describes the oscillations of two interacting particles in our double well [solid line in Fig. 1(d)].

To use our system as a fundamental building block of the Hubbard model, we must be able to prepare the particles in the ground state of the symmetric double well. To achieve this, we first initialize the system with both atoms in the left well and a tilt $\Delta \ll 0$. Then, we adiabatically change the tilt to bring the system into the ground state of the symmetric double well [29] (Sec. VI and Fig. S4 of [21]) [30].

To show that our fundamental building block already contains the physics that is responsible for the formation of ordered phases in a many-body system, we first measure the influence of the interaction energy $U$ on the distribution of the two particles between the wells. We therefore determine the probabilities $P_{1}=|\langle\Psi \mid L R\rangle|^{2}+|\langle\Psi \mid R L\rangle|^{2}$ of finding the two particles on different sites (single occupancy) and $P_{2}=|\langle\Psi \mid L L\rangle|^{2}+|\langle\Psi \mid R R\rangle|^{2}$ of finding both particles on the same site (double occupancy) by measuring the probabilities of having 0,1 , or 2 atoms in one of the wells (Sec. VII of [21]).

In a noninteracting system $(U=0)$, the spatial wave function of two particles in the ground state is an equal superposition of the basis states $|L L\rangle,|L R\rangle,|R L\rangle$, and $|R R\rangle$. This leads to equal probabilities $P_{1}$ and $P_{2}$ which we observe in our measurements [Fig. 3(a)].

In a system with strong repulsive on-site interactions, it is energetically unfavorable to have two atoms occupying the same site. The ground state of our system then becomes a two-particle analog to a Mott-insulating state which we observe as a reduction of double occupancy. For attractive interactions we observe an increase in double occupancy that marks the onset of a paired state. We interpret this state as the two-particle limit of the cold gas analog of a chargedensity-wave state as described in [31]. We perform these measurements for two different barrier heights. For the larger barrier height $(J / h \simeq 67 \mathrm{~Hz})$, we find good agreement with the prediction of the Hubbard model [Fig. 3(a)].
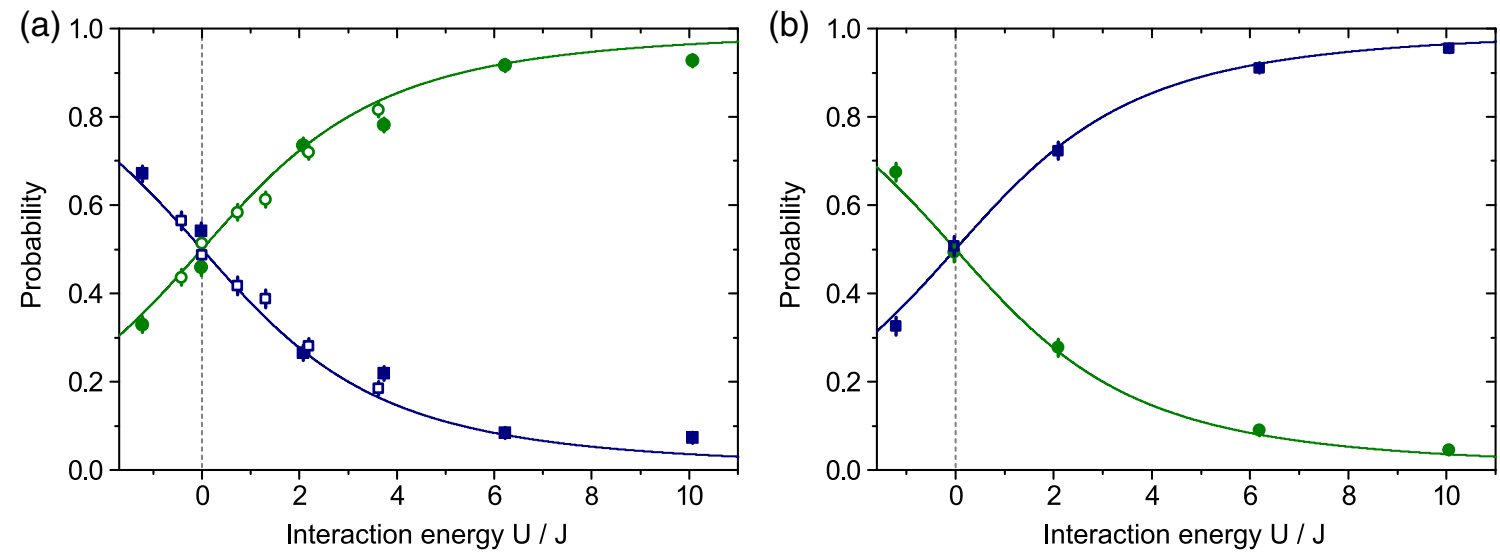

FIG. 3 (color online). Occupation statistics as a function of interaction strength. The relative probabilities of measuring both particles in the same well $\left(P_{2}\right.$, blue squares $)$ or in different wells $\left(P_{1}\right.$, green circles) are shown as a function of the on-site interaction energy $U$. Open (filled) symbols indicate a tunnel coupling of $J / h \simeq 142 \mathrm{~Hz}(J / h \simeq 67 \mathrm{~Hz})$, the solid lines show the prediction of the Hubbard model. (a) For the ground state $|a\rangle$, double occupancy is suppressed for increasing repulsive interactions. This indicates the crossover from a metallic to a Mott-insulating regime. For attractive interactions, double occupancy is enhanced, which we interpret as the onset of a charge-density-wave regime. (b) For the excited state $|c\rangle$, we observe the crossover to the charge-density-wave regime for strong repulsive interactions. For both measurements, the data have been corrected for the effect of the finite fidelities of preparation and detection (Sec. VII and Fig. S5 of [21]). The error bars denote the $1 \sigma$ statistical uncertainty. 


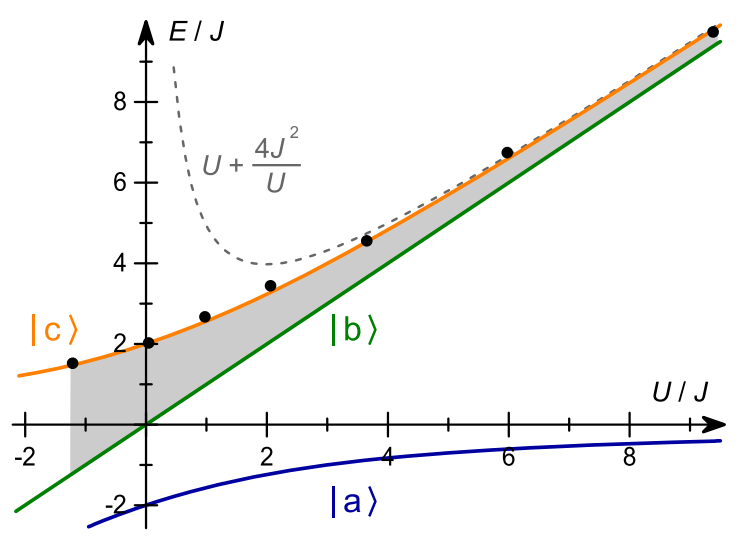

FIG. 4 (color online). Transition from first-order to secondorder tunneling. To directly measure the influence of the tunnel coupling on the energy of the system, we determine the energy difference $E_{b c}=E_{c}-E_{b}$ (shaded region) between state $|c\rangle$ (orange) and state $|b\rangle$ (green) using trap modulation spectroscopy. To compare our results to the eigenenergies of the Hubbard model [Fig. 1(b)], we plot the sum of $E_{b c}$ and the energy of state $|b\rangle\left(E_{b}=U\right)$. The error bars for the $1 \sigma$ statistical uncertainties (Sec. VIII of [21]) are smaller than the symbols of the data points. For $U=0$, we observe that $E_{b c} \approx 2 J$, which is consistent with single-particle tunneling. For increasing repulsion, first-order tunneling is suppressed and $E_{b c}$ converges to the superexchange energy $4 J^{2} / U$ (see dashed line).

For the smaller barrier height $(J / h \simeq 142 \mathrm{~Hz})$, we observe a small deviation from the theoretical expectations which might indicate that our system is approaching the limits of the Hubbard approximation.

Since in our isolated, two-particle system excited states are stable against relaxation, we can also prepare the system in the highest-energy eigenstate $|c\rangle$ (Fig. S4 of [21]). When measuring the occupation statistics of this state as a function of interaction strength, we find that the number of doubly occupied sites is enhanced for repulsive interactions [Fig. 3(b)]. This allows us to study the charge-density wave regime in a system with repulsive interactions.

In our final measurement, we directly probe the effect of the tunnel coupling on the energy of the system by trap modulation spectroscopy (Sec. VIII of [21]). For this, we initialize the system in state $|c\rangle$ and measure the energy difference to state $|b\rangle$ as a function of interaction strength. For $U=0$, the atoms are delocalized over the wells by single-particle tunneling. This leads to a change in the kinetic energy of the system that is proportional to the tunnel coupling $J$ (Fig. 4). As we increase the interactions, the system enters the insulating regime and first-order tunneling is suppressed. However, second-order tunneling is still possible and we observe a crossover to a new energy scale given by the superexchange energy $4 J^{2} / U$. This energy is directly responsible for the appearance of spin order in the ground state of the Hubbard model [32].

By combining a series of isolated double wells we can realize a dimerized lattice [13] where each dimer contains two fermions in a spin-singlet configuration with high fidelity. By adiabatically lowering the barriers between the double wells, we can then prepare a low-entropy state in a homogeneous lattice. Since our systems can be prepared with arbitrary filling factors they are also ideally suited to study the effects of hole doping. Additionally, the tunability of our potential allows us to explore finite-size lattices with arbitrary geometries [33] and introduce controlled disorder into our system. Finally, our experiments provide a starting point for scalable quantum computation with neutral atoms [34-37].

Supported by the European Research Council starting Grant No. 279697 and the Heidelberg Center for Quantum Dynamics.

S. M and A. B. contributed equally to this work.

* Corresponding author.

murmann@physi.uni-heidelberg.de

†resent address: MIT-Harvard Center for Ultracold Atoms, Research Laboratory of Electronics, and Department of Physics, Massachusetts Institute of Technology, Cambridge, Massachusetts 02139, USA.

[1] P. W. Anderson, Science 235, 1196 (1987).

[2] D. Jaksch, C. Bruder, J. I. Cirac, C. W. Gardiner, and P. Zoller, Phys. Rev. Lett. 81, 3108 (1998).

[3] R. Jördens, N. Strohmaier, K. Günter, H. Moritz, and T. Esslinger, Nature (London) 455, 204 (2008).

[4] U. Schneider, L. Hackermüller, S. Will, T. Best, I. Bloch, T. A. Costi, R. W. Helmes, D. Rasch, and A. Rosch, Science 322, 1520 (2008).

[5] W. S. Bakr, A. Peng, M. E. Tai, R. Ma, J. Simon, J. I. Gillen, S. Fölling, L. Pollet, and M. Greiner, Science 329, 547 (2010).

[6] J. F. Sherson, C. Weitenberg, M. Endres, M. Cheneau, I. Bloch, and S. Kuhr, Nature (London) 467, 68 (2010).

[7] T. Esslinger, Annu. Rev. Condens. Matter Phys. 1, 129 (2010).

[8] D. Pertot, A. Sheikhan, E. Cocchi, L. A. Miller, J. E. Bohn, M. Koschorreck, M. Köhl, and C. Kollath, Phys. Rev. Lett. 113, 170403 (2014).

[9] M. Greiner, O. Mandel, T. Esslinger, T. W. Hänsch, and I. Bloch, Nature (London) 415, 39 (2002).

[10] T. Stöferle, H. Moritz, C. Schori, M. Köhl, and T. Esslinger, Phys. Rev. Lett. 92, 130403 (2004).

[11] S. Fölling, S. Trotzky, P. Cheinet, M. Feld, R. Saers, A. Widera, T. Müller, and I. Bloch, Nature (London) 448, 1029 (2007).

[12] S. Trotzky, P. Cheinet, S. Fölling, M. Feld, U. Schnorrberger, A. M. Rey, A. Polkovnikov, E. A. Demler, M. D. Lukin, and I. Bloch, Science 319, 295 (2008).

[13] D. Greif, T. Uehlinger, G. Jotzu, L. Tarruell, and T. Esslinger, Science 340, 1307 (2013).

[14] R. A. Hart, P. M. Duarte, T.-L. Yang, X. Liu, T. Paiva, E. Khatami, R. T. Scalettar, N. Trivedi, D. A. Huse, and R. G. Hulet, arXiv:1407.5932.

[15] D. C. McKay and B. DeMarco, Rep. Prog. Phys. 74, 054401 (2011). 
[16] R. Jördens, L. Tarruell, D. Greif, T. Uehlinger, N. Strohmaier, H. Moritz, T. Esslinger, L. De Leo, C. Kollath, A. Georges, V. Scarola, L. Pollet, E. Burovski, E. Kozik, and M. Troyer, Phys. Rev. Lett. 104, 180401 (2010).

[17] F. Serwane, G. Zürn, T. Lompe, T. B. Ottenstein, A. N. Wenz, and S. Jochim, Science 332, 336 (2011).

[18] A. M. Kaufman, B. J. Lester, and C. A. Regal, Phys. Rev. X 2, 041014 (2012).

[19] A. M. Kaufman, B. J. Lester, C. M. Reynolds, M. L. Wall, M. Foss-Feig, K. R. A. Hazzard, A. M. Rey, and C. A. Regal, Science 345, 306 (2014).

[20] A. N. Wenz, G. Zürn, S. Murmann, I. Brouzos, T. Lompe, and S. Jochim, Science 342, 457 (2013).

[21] See Supplemental Material at http://link.aps.org/ supplemental/10.1103/PhysRevLett.114.080402 for details on the experimental setup, the experimental methods, and the data evaluation, which includes Refs. [22-26].

[22] F. Serwane, Ph.D. thesis, Ruperto-Carola-University of Heidelberg, 2011.

[23] M. Olshanii, Phys. Rev. Lett. 81, 938 (1998).

[24] Z. Idziaszek and T. Calarco, Phys. Rev. A 74, 022712 (2006).

[25] G. Zürn, F. Serwane, T. Lompe, A. N. Wenz, M. G. Ries, J. E. Bohn, and S. Jochim, Phys. Rev. Lett. 108, 075303 (2012).

[26] J. R. Petta, A. C. Johnson, J. M. Taylor, E. A. Laird, A. Yacoby, M. D. Lukin, C. M. Marcus, M. P. Hanson, and A. C. Gossard, Science 309, 2180 (2005).
[27] Note that this technique allows us to measure the atom number without parity projection.

[28] K. Winkler, G. Thalhammer, F. Lang, R. Grimm, J. Hecker Denschlag, A. J. Daley, A. Kantian, H. P. Büchler, and P. Zoller, Nature (London) 441, 853 (2006).

[29] J. R. Petta, A. C. Johnson, C. M. Marcus, M. P. Hanson, and A. C. Gossard, Phys. Rev. Lett. 93, 186802 (2004).

[30] We have verified that the probability of exciting atoms to higher trap levels while changing the potential is smaller than $1 \%$.

[31] A. F. Ho, M. A. Cazalilla, and T. Giamarchi, Phys. Rev. A 79, 033620 (2009).

[32] We cannot use trap modulation spectroscopy to directly measure the superexchange energy between the ground state $|a\rangle$ and state $|d\rangle$, since it does not couple to the spin of the particles.

[33] B. Zimmermann, T. Müller, J. Meineke, T. Esslinger, and H. Moritz, New J. Phys. 13, 043007 (2011).

[34] M. G. Raizen, S.-P. Wan, C. Zhang, and Q. Niu, Phys. Rev. A 80, 030302 (2009).

[35] M. Anderlini, P. J. Lee, B. L. Brown, J. Sebby-Strabley, W. D. Phillips, and J. V. Porto, Nature (London) 448, 452 (2007).

[36] L. Isenhower, E. Urban, X. L. Zhang, A. T. Gill, T. Henage, T. A. Johnson, T. G. Walker, and M. Saffman, Phys. Rev. Lett. 104, 010503 (2010).

[37] T. Wilk, A. Gaëtan, C. Evellin, J. Wolters, Y. Miroshnychenko, P. Grangier, and A. Browaeys, Phys. Rev. Lett. 104, 010502 (2010). 